\title{
Receptivity of Hypersonic Boundary Layers over Straight and Flared Cones
}

\author{
P. Balakumar ${ }^{*}$ and Michael A. Kegerise ${ }^{* *}$ \\ Flow Physics and Control Branch \\ NASA Langley Research Center, Hampton, VA 23581
}

\begin{abstract}
The effects of adverse pressure gradients on the receptivity and stability of hypersonic boundary layers were numerically investigated. Simulations were performed for boundary layer flows over a straight cone and two flared cones. The steady and the unsteady flow fields were obtained by solving the twodimensional Navier-Stokes equations in axi-symmetric coordinates using the $5^{\text {th }}$-order accurate weighted essentially non-oscillatory (WENO) scheme for space discretization and using third-order total-variation-diminishing (TVD) Runge-Kutta scheme for time integration. The mean boundary layer profiles were analyzed using local stability and non-local parabolized stability equations (PSE) methods. After the most amplified disturbances were identified, two-dimensional plane acoustic waves were introduced at the outer boundary of the computational domain and time accurate simulations were performed. The adverse pressure gradient was found to affect the boundary layer stability in two important ways. Firstly, the frequency of the most amplified second-mode disturbance was increased relative to the zero pressure gradient case. Secondly, the amplification of first- and second-mode disturbances was increased. Although an adverse pressure gradient enhances instability wave growth rates, small nose-tip bluntness was found to delay transition due to the low receptivity coefficient and the resulting weak initial amplitude of the instability waves. The computed and measured amplitudefrequency spectrums in all three cases agree very well in terms of frequency and the shape except for the amplitude.
\end{abstract}

\section{Introduction}

Accurate prediction of transition onset and transition end points, as well as modeling of the transitional and turbulent regions, are major concerns when using CFD codes to compute aerodynamic quantities. Our understanding of different instability mechanisms, and of different transition processes in shear layers, have greatly improved in the last several decades. ${ }^{1-8}$ Transition-prediction methods, however, have not made much progress ${ }^{9}$. The main difficulty is due to the nature of the transition process itself, which depends on the boundary layer characteristics and on the frequency and wave number distributions of the disturbances that enter the boundary layer. While laminar boundary layer profiles can be computed easily, the computation, prediction or prescription of the initial disturbance distributions inside the boundary layer is difficult. These disturbances, which are generated by unsteady freestream disturbances or by the interaction between freestream disturbances and surface roughness, are stochastic in nature and are difficult to quantify in general. Therefore, in any new transition prediction strategy, these disturbances should be quantified with the minimum amount of information necessary to predict the transition onset accurately. The objectives of this research work are to address some of these issues and to eventually improve transition-prediction methods.

The most popular transition prediction method is the physics based $\mathrm{e}^{\mathrm{N}}$ correlation method. This method is easy to implement and yields satisfactory results if appropriate N-Factors are used to fix the transition onset. This number depends on several parameters including geometry, tunnel noise and surface roughness. For a particular case, the practice is to choose transition onset N-Factors that are based on previous correlations for similar conditions. One example illustrating the difficulty in predicting transition using this simple method is shown in Fig. 1 (from Horvath et al. ${ }^{10}$ ). Here, the transition onset locations obtained over a $5^{\circ}$ flared cone under different tunnel noise conditions is shown. The measurements were performed in the NASA LaRC Mach 6 Quiet Tunnel under low and high noise levels, and in the NASA LaRC 20 inch Mach 6 Tunnel, which is a conventional tunnel with a high freestream noise

\footnotetext{
${ }^{*}$ Research Scientist, Member AIAA

${ }^{* *}$ Research Scientist, Member AIAA
} 
level. The measured transition onset locations were at 16,10 and 12 inches for the three cases respectively, and the corresponding computed N-Factors at transition onset were 7.8, 3.0 and 3.8 respectively. The reason for this scatter is mainly attributed to the different freestream noise levels that exist in the different tunnels or at different test conditions. Therefore, a rational approach to improve the prediction capability is to include the effects of freestream acoustic disturbances on the boundary layer in the prediction methods.

In our previous work, ${ }^{11-14}$ we have investigated the interaction of forced slow and fast acoustic waves with hypersonic boundary layers over sharp and blunt flat plates, wedges and cones. The results showed that for flows over sharp-nose cases, the instability waves are generated very close to the leading edge and the receptivity coefficient is about 5-10 times the amplitude of the forced slow acoustic wave. The amplitude of the instability waves generated by slow acoustic waves was found to be about 20 times larger than those generated by fast acoustic waves. The results also revealed that bluntness and wall cooling stabilize the first mode and produce instability waves with an initial amplitude that is orders of magnitude smaller than the amplitude of the forced acoustic waves.

In this paper, the effects of an adverse pressure gradient on the receptivity, stability and transition of hypersonic boundary layers are investigated. To that end, we continue our previous approach in which the amplitudes of the disturbances inside the boundary layer, generated by freestream acoustic disturbances, are computed. Simulations were performed on three axisymmetric geometries for which experimentally measured instability data are available for comparison: 1) a straight cone, 2) a circular flared cone with the flare starting from the nose, and 3) a circular flared cone with an initial straight nose region tangent to a flare downstream. The first two geometries were tested in the Boeing/AFOSR Mach 6 Quiet Tunnel at Purdue University ${ }^{15-16}$ and the third geometry was tested in the NASA Langley Mach 6 Quiet Tunnel. ${ }^{17-18}$ In each case, we first solved for the mean flow field and then performed linear local and non-local stability analyses for the computed boundary-layer profiles. After the parameters for the most amplified disturbances were identified, two-dimensional acoustic disturbances were superimposed on the outer boundary of the computational domain and time accurate simulations were performed. The results of the simulations were then compared to the available experimental data.

\section{Models and Flow Conditions}

The first model is a $7^{\circ}$ half-angle sharp-tipped cone with a nose radius of $0.0125 \mathrm{~mm}$ (Fig. 2). Computations were performed for a freestream Mach number of 6 . The freestream stagnation temperature and pressure were $430^{\circ} \mathrm{K}$ and $12.2 \mathrm{kPa}(140 \mathrm{psi})$, yielding a unit Reynolds number of $10.4 * 10^{6} / \mathrm{m}$. The simulations were performed for a constant wall temperature of $300^{\circ} \mathrm{K}$. This model geometry was tested in the Boeing/AFOSR Mach 6 Quiet Tunnel at the same freestream conditions. ${ }^{15-16}$

The second model is a circular flared cone with a spherical blunt nose region (Fig. 3). The flared region is joined to the blunt nose very near the tip. The concave circular flare has a radius of $3.0 \mathrm{~m}$ and the nose has a radius of 1.0 $\mathrm{mm}$. At the juncture, the flare and the nose have a slope of 2 degrees. Computations were performed for a freestream Mach number of 6 . The freestream stagnation temperature and pressure were $433^{\circ} \mathrm{K}$ and $12.2 \mathrm{kPa}(140 \mathrm{psi})$, yielding a unit Reynolds number of $10.3 * 10^{6} / \mathrm{m}$. The simulations were also performed for a constant wall temperature of $300^{\circ} \mathrm{K}$. As with the previous model, this geometry was tested in the Boeing/AFOSR Mach 6 Quiet Tunnel at the same freestream conditions. ${ }^{15}$

The third model consists of a $5^{\circ}$ half-angle sharp-cone region and a circular flared-cone region (Fig. 4). The model is $50.8 \mathrm{~cm}$ (20 in.) long with a $25.4 \mathrm{~cm}$ (10 in.) front straight-cone region that is connected to a circular flared-cone with a radius of $2.36 \mathrm{~m}$ (93.07 in.). At the juncture, the straight cone and the flared cone have the same slope. Computations were performed for a freestream Mach number of 6 . The freestream stagnation temperature and pressure were $400^{\circ} \mathrm{K}\left(810^{\circ} \mathrm{R}\right)$ and $11.33 \mathrm{kPa}(130 \mathrm{psi})$, yielding a unit Reynolds number of $8.950^{*} 10^{6} / \mathrm{m}$ $\left(2.727 * 10^{6} / \mathrm{ft}\right)$. The simulations were performed for an adiabatic wall condition. This model geometry was tested in the NASA Langley Mach 6 Quiet Tunnel. ${ }^{17-18}$ 


\section{Governing Equations}

The governing equations are the two-dimensional unsteady compressible Navier-Stokes equations, written in conservation form and in cylindrical coordinates:

$$
\begin{gathered}
\frac{\partial}{\partial t} r Q+\frac{\partial r F}{\partial x}+\frac{\partial r G}{\partial r}=S, \\
Q=\left[\begin{array}{l}
\rho \\
\rho E \\
\rho u \\
\rho v
\end{array}\right] .
\end{gathered}
$$

Here, $(x, r)$ are the cylindrical coordinates, $(u, v)$ are the velocity components, $\rho$ is the density, and $p$ is the pressure. The total energy, $E$, is given by:

$$
\begin{gathered}
E=e+\frac{u^{2}+v^{2}}{2}, \\
e=c_{v} T, p=\rho R T,
\end{gathered}
$$

where $e$ is the internal energy and $T$ is the temperature. The fluxes $F, G$ and the source term $S$ are described in Kara et al. ${ }^{12}$ The viscosity, $\mu$, is computed using Sutherland's law and the thermal conductivity, $k$, is given in terms of the Prandtl number, $P r$. The variables $\rho, p, T$ and velocity are non-dimensionalized by their corresponding reference variables $\rho_{\infty}, p_{\infty}, T_{\propto}$ and $\sqrt{R T_{\infty}}$ respectively. The reference value for length is given by $\sqrt{v x_{0} / U_{\infty}}$, where $x_{0}$ is a reference location. For the computations, the equations were transformed from the physical coordinate system $(x, r)$ to the computational curvilinear coordinate system $(\xi, \eta)$ in a conservative manner.

\section{A. Solution Algorithm}

The governing equations were solved using a $5^{\text {th }}$-order accurate weighted essentially non-oscillatory (WENO) scheme for space discretization and a $3^{\text {rd }}$-order total-variation-diminishing (TVD) Runge-Kutta scheme for time integration. The WENO and TVD methods and formulas are explained in Shu. ${ }^{19}$ The application of the ENO method to the Navier-Stokes equations is presented by Atkins. ${ }^{20}$ The solution method implemented in the present computations is described in Balakumar. ${ }^{21}$

Schematic diagrams of the computational setup for each model case are shown in Figs. 2-4. The outer boundary of the computational domain lies outside the shock and follows a parabola so that the boundary layer growth was accurately captured. At the outflow boundary of the computational domain, an extrapolation boundary condition was used. At the wall, the simulations employed viscous conditions for the velocities and an adiabatic or constant wall temperature. The density was computed from the continuity equation. In the mean-flow computations, the free-stream values at the outer boundary were prescribed. In all cases, the model centerline was aligned with the freestream flow. In the unsteady computations, acoustic perturbations were superimposed on the uniform mean flow at the outer boundary of the computational domain.

The solution procedure was to first compute the steady mean flow by performing unsteady computations using a variable time step until the maximum residual reaches a small value $\left(\sim 10^{-11}\right)$. For those computations, a CFL number of 0.2 was used. The next step was to introduce an unsteady acoustic disturbance at the outer boundary of the computational domain with the following form:

$$
p_{a c}(x, t)=\tilde{p}_{a c} e^{i\left(\alpha_{a c} x-\omega t\right)},
$$


$48^{\text {th }}$ AIAA Aerosciences Meeting, January 4-7, 2010, Orlando, Florida

where $\alpha_{a c}$ is the $x$ wave number of the acoustic disturbance and $\omega$ is the corresponding frequency. Then, time accurate computations were performed to investigate the interaction of these disturbances with the boundary layer and their subsequent downstream evolution. The computational grid, which was generated using analytical formulae, stretches in the $\eta$ direction close to the wall and is uniform outside of the boundary layer. In the $\xi$ direction, the grid was very fine near the nose and then uniform in the region downstream.

\section{A. $7^{\circ}$ Half-Angle Sharp-Tipped Cone}

\section{Results}

\section{Mean Flow}

Figure 5 presents the computed mean density contours for the sharp-tipped cone. In Fig. 5a, the flow field for the entire computational domain is shown, while Fig. 5b shows a close-up view of the nose region. The mean surface pressure distribution along the axial direction of the cone is depicted in Fig. 6a, and as expected, the pressure gradient is zero except for a region very close to the nose tip. Figure $6 \mathrm{~b}$ presents the boundary layer density profiles at different axial locations in similarity coordinates. The compressible Blasius similarity profile is also included for comparison. In this figure, the boundary layer profiles are observed to slowly approach the Blasius similarity profile, and by $x=3.0 \mathrm{~cm}$, the boundary layer profiles are essentially equal to the similarity profile.

\section{Linear Stability}

Linear stability analysis and the PSE analysis were performed on the computed boundary layer profiles for the sharp-tipped cone boundary layer. The results are shown in Fig. 7, where the computed N-factor curves obtained from the linear stability analysis and the PSE calculations are plotted. For the PSE results in particular, the presented N-Factors were obtained using $(\rho u)_{\max }$. The two axial locations annotated in the figure at $x=208$ and 490 $\mathrm{mm}$, correspond to those where measured wall-pressure fluctuation data are available for comparison. ${ }^{16}$ At these locations the maximum $\mathrm{N}$-factors are 3.5 and 7.0 respectively, and the corresponding frequencies are 350 and 220 $\mathrm{kHz}$.

\section{Interaction of Slow Acoustic Waves with the sharp-tipped cone}

After the mean flow was computed, two-dimensional slow acoustic disturbances (phase speed equal to $U_{\infty}-c_{\infty}$ ) were introduced at the outer boundary of the computational domain and time-accurate simulations were performed. The amplitude of these forced acoustic disturbances was given a small value of $\tilde{p}_{a c} / p_{\infty}=2 * 10^{-5}$ to ensure that the disturbances evolving in the boundary layer remained in the linear regime. This allowed the results of the computations to be compared to linear PSE calculations. Several frequencies were considered in the simulations, but here, we present the results for a frequency of $216 \mathrm{kHz}$, which corresponds to the maximum $\mathrm{N}$-factor at $x=490$ $\mathrm{mm}$. Figures 8, 9, and 10 show the results for the evolution of unsteady fluctuations produced by the slow acoustic wave at a fixed time. Contours of the density fluctuations in the entire computational domain are shown in Fig. 8. Here, the perturbation field can be divided into four regions. The first region is the area outside the shock, where the acoustic waves propagate uniformly. The second region is the shock layer across which acoustic waves are transmitted. The third region is the area between the shock and the boundary layer, which consists of the transmitted external acoustic field and disturbances that are radiated from the boundary layer. The fourth region is the boundary layer where instability waves evolve in the downstream direction. Figure 9 provides more detailed views of the perturbation field in the nose region (Fig. 9a), in the tip region (Fig. 9b), and near the end of the computational domain (Fig. 9c). In the tip region, we see that the freestream acoustic disturbances penetrate the shock layer and transform smoothly to boundary layer instability waves, which have a wavelength that is comparable to the wavelength of the acoustic disturbance. Moving further downstream (Figs. 9a and 9c), we see that the penetration of freestream disturbances across the shock layer is diminished, and in the region between the shock and the boundary layer, the disturbance levels are very low. This suggests that the freestream acoustic disturbances do not excite the boundary layer downstream of the cone tip; instead, the boundary layer disturbances originate from the nose region of the cone.

Figure 10 shows the evolution of the wall pressure fluctuations on the cone that are associated with the acoustically driven boundary layer instabilities. In Fig. 10a, the amplitude of the wall pressure fluctuations is 
plotted on a linear scale, while Fig. 10b depicts the results on a log scale. The initial amplitude of the wall-pressure fluctuation, very close to the tip $(x \approx 0)$, is observed to be approximately equal to the amplitude of the freestream acoustic level. The disturbance growth over the first $25 \mathrm{~cm}$ of the cone, which is associated with the first mode, is observed to be very weak. As the disturbances transform to second mode with increasing downstream distance, the disturbance grows exponentially as expected. The results of linear PSE calculations for the same mean boundary layer profile are included in Fig. 10b for comparison with the computations. The PSE results are observed to agree very well with the computations. This agreement is also further evidence that the boundary-layer disturbances originate from a small region near the cone tip since the PSE calculations were performed with a disturbance input at a single axial location.

A receptivity coefficient was defined as the amplitude of the wall-pressure fluctuations at the neutral point nondimensionalized by the freestream acoustic pressure:

$$
C_{\text {recpt }, p_{\text {wall }}}=\frac{\left(p_{\text {wall }}\right)_{n}}{p_{a c}}
$$

The amplitude of the wall-pressure fluctuation at the neutral point was estimated by following the PSE results up to the neutral point. With that value, and a freestream acoustic level of $2 * 10^{-5}$, the receptivity coefficient is approximately 7.0 for a frequency of $216 \mathrm{kHz}$.

Figure 11 presents a comparison between the computed and the measured amplitudes of the wall-pressure fluctuations at two axial locations $(x=208$ and $490 \mathrm{~mm})$. Recall that the simulations were performed for freestream acoustic disturbances over a range of frequencies, so the amplitude data are presented as a function of frequency. Also in the computations, the amplitude of the acoustic disturbances was set to $\tilde{p}_{a c} / p_{\infty}=2 * 10^{-5}$. The actual amplitude of the freestream acoustic disturbances present in the experimental measurements is unknown. Figure 11a compares the computed wall-pressure amplitude at $x=208 \mathrm{~mm}, R e / \mathrm{m}=10.4^{*} 10^{6}$ and the wall-pressure amplitude spectrum measured at the same axial location and flow condition by Casper et al. ${ }^{16}$ Note that the pressure spectrum shown in Fig. 11a was measured under noisy-flow conditions because under quiet-flow conditions, the wall-pressure fluctuations were below the noise floor of the pressure transducer. Also, the transducer used for this measurement had a sensor size of approximately $1 \mathrm{~mm}$, which is on the order of the instability wavelengths $(2.4$ to $3.5 \mathrm{~mm}$ ) in the boundary layer. Since the effect of spatial averaging on the sensor dynamic response was not characterized in the experiment, the measured spectrum amplitude shown in the plot is qualitative. Nevertheless, the frequencies and shape of the computed amplitude spectrum compare favorably with the experimental data. Figure $11 \mathrm{~b}$ presents a similar comparison between the computational and experimental wall-pressure spectrum at $x=490$ $\mathrm{mm}, R e / \mathrm{m}=10.4 * 10^{6}$. This experimental data was obtained under quiet-flow conditions with the same pressure transducer. As before, the frequencies and shape of the computed wall-pressure spectrum agree very well with the measurements. Although the spectral comparisons made here are qualitative in amplitude, the computational approach presented in this paper should be able to quantitatively predict the amplitude of the instability waves provided we know the actual amplitude of the freestream acoustic disturbances that were present in the experiment.

\section{B. Circular Flared Cone with Spherical Blunt Nose}

\section{Mean Flow}

Figure 12 presents the computed mean density contours for the circular flared cone with a spherical blunt nose region. In Fig. 12a, the flow field for the entire computational domain is shown, while Fig. 12b shows a close-up view of the nose region. The leading edge shock and the compression waves formed by the circular flare are clearly observed in the figures. The mean surface pressure distribution along the axial direction of the flared cone is depicted in Fig. 6a, and as expected, the flare introduces an adverse pressure gradient along the surface, starting from the tip. The mean density and velocity profiles in the boundary layer at several different axial locations are shown in Figs. 13a and 13b, respectively. Beyond an axial location of approximately $5 \mathrm{~cm}$, the boundary layer thickness is observed to remain nearly constant at a thickness of $0.12 \mathrm{~cm}$ : a consequence of the balancing forces generated by the concave curvature of the flared-cone surface. It is also noted from Fig. 13b that the velocity profiles change slowly along the axial direction. 


\section{Linear Stability}

Linear local stability analysis and non-local PSE calculations were performed on the computed mean velocity profiles of the circular flared cone. The results are shown in Fig. 14, where the computed N-factor curves obtained from the linear stability analysis (Fig. 14a) and the PSE calculations (Fig. 14b) are plotted. For the PSE results in particular, the presented N-Factors were obtained using $(\rho u)_{\max }$; however, N-Factors obtained with other variables were not significantly different. The measured wall-pressure fluctuations, to which the present computational results are compared, were obtained in the experiments by Wheaton et al..$^{15}$ at an axial location of $x=400 \mathrm{~mm}$. From Fig. 14, the maximum N-Factors at that axial location are about 13.0 for both analysis methods, and the most amplified frequencies are about $279.0 \mathrm{kHz}$ (linear stability) and $286.0 \mathrm{kHz}$ (PSE). Note that even with these large $\mathrm{N}$-Factors, transition was not observed on the flared-cone model in the experiment. ${ }^{15}$

\section{Interaction of Acoustic Waves with the flared cone}

As in the previous case, after the mean flow was computed, two-dimensional acoustic disturbances (with an amplitude of $\left.\tilde{p}_{a c} / p_{\infty}=2 * 10^{-6}\right)$ were introduced at the outer boundary of the computational domain and timeaccurate simulations were performed. These simulations included both slow and fast (phase speed equal to $U_{\infty}+c_{\infty}$ ) acoustic disturbances for a range of frequencies. Here, we present the results for a frequency of $292.5 \mathrm{kHz}$. Figures 15,16 , and 17 show the results for the evolution of unsteady fluctuations produced by the freestream acoustic disturbances at a fixed time. Contours of the density fluctuations for the slow acoustic wave are shown in Fig. 15 for the entire computational domain (Fig. 15a), the nose region (Fig. 15b), and the tip region (Fig. 15c) of the circular flared cone. The perturbation field generated by the acoustic disturbance is qualitatively similar to that for the sharp-tipped cone and again, we observe that boundary-layer disturbances originate from the nose region of the cone.

Figure 16 shows the evolution of the wall-pressure fluctuations on the flared cone that are induced by the slow acoustic wave. In Fig. 16a, the amplitude of the wall-pressure fluctuation is plotted on a linear scale, while Fig. 16b depicts the results on a log scale. The figures clearly show the generation and the eventual exponential growth of the instability waves inside the boundary layer. However, an important difference between the blunt flared cone and the straight sharp-tipped cone (Fig. 10) is the evolution of the first mode near the nose region. For the sharp-tipped straight cone, the first mode grows slightly in the downstream direction due to non-parallel effects in the boundary layer. However, for the blunt flared-cone case, the first mode decreases up to $70 \mathrm{~mm}$ and then remains constant up to $150 \mathrm{~mm}$ before smoothly transforming to the second mode, with subsequent exponential growth in the downstream direction. This behavior is linked to the entropy layer produced by the large bluntness of the flared cone geometry (Reynolds number of 10400 based on nose radius). Another important difference between the straight sharp-tipped and blunt flared cone results is the growth rate of the instability waves, which is larger for the flared cone due to the adverse pressure gradient. Figure 17 shows the evolution of the wall pressure fluctuations on the flared cone that are associated with the fast acoustic wave. In this case, the behavior is similar to that for the slow acoustic wave. However, the decay of the first mode is less than that for the slow acoustic wave. Therefore, the disturbances induced by the fast acoustic wave have higher amplitudes than those induced by a slow acoustic wave. In particular, the amplitude of the wall-pressure fluctuations at $x=40 \mathrm{~cm}$ are 0.0028 and 0.0055 for the slow and fast acoustic waves, respectively. This amplitude difference was previously observed in computations for hypersonic flows over blunt cones. ${ }^{13}$ The results of PSE calculations for the flared cone boundary layer are included in Figs. $16 \mathrm{~b}$ and $17 \mathrm{~b}$ for comparison to the computations. The computed disturbance growth, for both slow and fast acoustic waves, is observed to agree very well with the PSE results.

As before, a receptivity coefficient was defined as the amplitude of the wall-pressure fluctuations at the neutral point non-dimensionalized by the freestream acoustic pressure, and the amplitude of the wall-pressure fluctuation at the neutral point was estimated by following the PSE results up to the neutral point. The receptivity coefficients for the slow and fast acoustic waves, at a frequency of $292.5 \mathrm{kHz}$, were found to be $4.9^{*} 10^{-3}$ and $9.3 * 10^{-3}$, respectively. These coefficients are much smaller than the receptivity coefficient of 7.0 for the sharp-tipped cone. This result agrees with our earlier receptivity calculations for sharp and blunt circular cones in hypersonic flows. ${ }^{12-13}$

Figure 18 presents a comparison between the computed and the measured amplitudes of the wall-pressure fluctuations at an axial location of $x=40 \mathrm{~cm}$. Here, the results for the computations correspond to fast acoustic waves over a range of frequencies. The measured wall-pressure spectrum was obtained in the experiment by Wheaton et al. ${ }^{15}$ As with the sharp-tipped cone, the frequencies and the shape of the computed amplitude spectrum compare favorably with the experimental data. In comparing amplitudes, however, the results are qualitative since 
$48^{\text {th }}$ AIAA Aerosciences Meeting, January 4-7, 2010, Orlando, Florida

the actual amplitude of the freestream acoustic disturbances that were present in the experiment are unknown and the measured wall-pressure spectrum was obtained with an uncalibrated pressure transducer.

\section{Flared-Cone with a sharp-tipped nose}

\section{Mean Flow and Linear Stability}

Details for the computed mean flow and linear stability analysis for the sharp-tipped flared-cone model are presented in Balakumar and Malik ${ }^{22}$ and Horvath et al. ${ }^{10}$ Numerical simulation of the stability and receptivity to fast acoustic disturbances over a blunt cone is presented in Zhong. ${ }^{23}$ Figure 19 presents the computed mean density contours for this geometry. The leading edge shock and the compression waves generated by the circular flare, which starts at $x=25 \mathrm{~cm}$, are clearly observed in the figure. The mean surface pressure distribution along the axial direction of the cone is depicted in Fig. 6a, and as expected, the adverse pressure gradient starts at the flared region of the model. The most amplified frequency for this model geometry, as determined by the linear stability analysis, was found to be $220 \mathrm{kHz}$.

\section{Interaction of Acoustic Waves with the Flared Cone}

With the mean flow computed, two-dimensional acoustic disturbances were introduced at the outer boundary of the computational domain and time-accurate simulations were performed. These simulations included both slow and fast acoustic waves for a range of frequencies. Here, we present the results for the most amplified frequency of $220 \mathrm{kHz}$. Contours of the density fluctuations for the slow acoustic wave (with an amplitude of $\tilde{p}_{a c} / p_{\infty}=2 * 10^{-6}$ ) are shown in Fig. 20 for the entire computational domain (Fig. 20a) and the nose region (Fig. 20b). The perturbation field generated by the acoustic disturbance is qualitatively similar to that for the other two model cases and again, we observe that boundary-layer disturbances originate from the nose region of the cone.

Figure 21 shows the evolution of the wall-pressure fluctuations on the flared cone that are induced by the slow acoustic wave. Here, data are shown for two freestream acoustic amplitude levels equal to $2 * 10^{-5}$ and $2 * 10^{-6}$. Similar to the sharp-tipped cone, the amplitude of the boundary-layer disturbances near the tip $(x \sim 0)$ is equal to the freestream acoustic amplitude. As expected, the disturbances are observed to grow from the tip region and then grow exponentially through the flared region of the cone. In comparison to the sharp-tipped cone case, the disturbance growth rate for the flared-cone model is significantly higher due to the adverse pressure gradient. In fact, the large growth rate leads to non-linear saturation at a pressure level of $\tilde{p}_{w} / p_{\infty} \sim 1.0$. It is interesting to note that the point at which saturation occurs increases by only $3 \mathrm{~cm}$ when the freestream acoustic amplitude is decreased by an order of magnitude. This small difference is a result of the large disturbance growth rate due to the adverse pressure gradient in the flared region of the cone. Experimental measurements on this flared cone model geometry (in the NASA Langley Mach 6 Quiet Tunnel) by Blanchard ${ }^{17}$ and Lachowicz et al. ${ }^{18}$ found transition to occur around $40 \mathrm{~cm}$. This is close to the axial location where non-linear saturation of the boundary layer instabilities was observed in the present computations.

The receptivity coefficient for this model geometry was about 5-7 times the freestream acoustic level, which is similar to that calculated for the $7^{\circ}$ half-angle sharp-tipped cone. Given that the geometry of the nose region is similar for the two cases, and the fact that boundary layer instabilities originate in the nose region, the correspondence between the receptivity coefficients is expected.

Figure 22 presents a comparison between the computed spectrum of maximum mass fluctuations $(\rho u)_{\max }$ and the measured disturbance spectrum in the boundary layer at an axial location of $x=40 \mathrm{~cm}$. Here, the results for the computations correspond to slow acoustic waves over a range of frequencies. The experimental data is an uncalibrated hot-wire voltage spectrum measured at the peak location in the boundary layer disturbance profile. ${ }^{18}$ The peak frequency for the computed amplitude spectrum is $220 \mathrm{kHz}$, which compares well with the measured peak frequency of $226 \mathrm{kHz}$.

\section{Conclusions}

Numerical simulations were performed to study the effects of adverse pressure gradients on the receptivity and stability of hypersonic boundary layers. These simulations were performed on three axisymmetric geometries for which experimentally measured instability data are available for comparison: 1) a 7-degree, sharp-tipped cone, 2) a circular flared cone with a blunt tip, and 3) a flared cone comprised of a 5-degree sharp-tipped straight section and 
an aft circular-flare region. For each case, the mean flow was obtained by solving the two-dimensional NavierStokes equations in axisymmetric coordinates using the $5^{\text {th }}$-order accurate weighted essentially non-oscillatory (WENO) scheme for space discretization and the $3^{\text {rd }}$-order total-variation-diminishing (TVD) Runge-Kutta scheme for time integration. Local stability analysis and PSE calculations were then applied to the computed mean boundary-layer profiles to identify the most amplified disturbances. Then, two-dimensional plane acoustic waves were introduced at the outer boundary of the computational domain and time-accurate simulations were performed.

In general, the computations indicate that the receptivity of the boundary layer to freestream acoustic disturbances is confined to the nose region for each of the cone geometries considered. Furthermore, the receptivity coefficient for the sharp tipped cone geometries was found to be several orders of magnitude larger than the circular flared cone geometry with a blunt tip. This bluntness effect is in agreement with previous studies of hypersonic boundary layer receptivity. ${ }^{12,13}$ The presence of an adverse pressure gradient was found to affect boundary layer stability in two important ways. Firstly, the frequency of the most amplified second-mode disturbance was shifted to a higher value relative to that for a straight cone, which has a zero pressure gradient. Secondly, the amplification of first- and second-mode disturbances is increased by the adverse pressure gradient. It is interesting to note that transition did not occur on the circular flared cone geometry in the experiments by Wheaton et al., ${ }^{15}$ in spite of the enhanced instability wave growth rate resulting from the adverse pressure gradient. This is probably due to the very low receptivity coefficient associated with the cone bluntness, which results in weak initial amplitudes for the instability waves. Finally, the computed and measured wall-pressure spectrums agreed in terms of frequency and spectral shape of the most amplified boundary-layer disturbance. However, to quantitatively predict the amplitude of the wall-pressure fluctuation with the present computational approach, a measurement of the freestream acoustic spectrum is required. Nevertheless, the present work represents a rational approach in which the effects of freestream acoustic disturbances are included in the analysis, and that will ultimately lead to improved predictions of boundary layer transition.

\section{References}

${ }^{1}$ Mack, M.L., “Boundary Layer Stability Theory,” JPL Report No. 900-277 Rev. A, Pasadena, CA, Nov. 1969.

${ }^{2}$ Kendall,J. M., "Wind Tunnel Experiments Relating to Supersonic and Hypersonic Boundary-Layer Transition ," AIAA J., Vol. 13, No. 3, 1975, pp. 290-299.

${ }^{3}$ Stetson, K. F., and Kimmel, R. L., “On Hypersonic Boundary-Layer Stability,” AIAA Paper 92-0737, January 1992.

${ }^{4}$ Maslov, A. A., Shiplyuk, A. N., Sidorenko, A. A and Arnal, D., "Leading Edge Receptivity of a Hypersonic Boundary layer on a Flat Plate," J. Fluid Mech., Vol. 426, 2001, pp. 73-94.

${ }^{5}$ Fedorov, A. V., and Khokhlov, A. P., “ Excitation of Unstable Modes in a Supersonic Boundary Layer by Acoustic Waves," Fluid Dynamics, Vol. 26, No. 4, pp. 531-537, July 1991.

${ }^{6}$ Fedorov, A.V., "Receptivity of a High-Speed Boundary Layer to Acoustic Disturbances," Journal of Fluid Mechanics, Vol. 491, 2003, pp. 101-129.

${ }^{7}$ Ma, Y. and Zhong, X., "Receptivity of a Supersonic Boundary Layer over a Flat Plate. Part 1. Wave Structures and Interactions," Journal of Fluid Mechanics, Vol. 488, 2003, pp. 31-78.

${ }^{8}$ Ma, Y. and Zhong, X., "Receptivity of a Supersonic Boundary Layer over a Flat Plate. Part 2. Receptivity to Freestream Sound," Journal of Fluid Mechanics, Vol. 488, 2003, pp. 79-121.

${ }^{9}$ Malik, M. R., "Boundary-Layer Transition Prediction Toolkit,", AIAA paper 1997-1904.

${ }^{10}$ Horvath, T.J., Berry, S.A., Hollis, B.R., Chang, C.L., and Singer, B.A., "Boundary Layer Transition on Slender Cones in Conventional and Low Disturbance Mach 6 Wind Tunnels," AIAA Paper 2002-2743, June 2002.

${ }^{11}$ Malik, M.R. and Balakumar, P., "Instability and Transition in Three-Dimensional Supersonic Boundary Layers," AIAA Paper 1992-5049, December 1992.

${ }^{12}$ Kara, K., Balakumar, P., and Kandil, O.A., "Receptivity of Hypersonic Boundary Layers Due to Acoustic Disturbances over Blunt Cone," AIAA Paper 2007-945, Jan. 2007.

${ }^{13}$ Kara, K., Balakumar, P., and Kandil, O.A., "Effects of Nose Bluntness on Stability of Hypersonic Boundary Layers over a Blunt Cone," AIAA Paper 2007-4492, June 2007.

${ }^{14}$ Kara, K., Balakumar, P., and Kandil, O.A., "Effects of Wall Cooling on Hypersonic Boundary Layer Receptivity over a Cone," AIAA Paper 2008-3734, June 2008.

${ }^{15}$ Wheaton, B., Juliano, T.J., Berridge, D.C., Chou, A., Gilbert, P.L., Casper, K.M., Steen, L.E., Schneider, S., and Johnson, H.B., "Instability and Transition Measurements in the Mach 6 Quiet Tunnel," AIAA Paper 2009-3559, June 2009.

${ }^{16}$ Casper, K.M., Beresh, S.J., Henfling, J.F., Spillers, R.W., Pruett, B., and Schneider, S.P., "Hypersonic Wind-Tunnel Measurements of Boundary-Layer Pressure Fluctuations,” AIAA Paper 2009-4054, Jun 2009.

${ }^{17}$ Blanchard, A.E., An investigation of Wall-Cooling Effects on Hypersonic Boundary-Layer Stability in a Quiet Wind Tunnel, Ph.D. Dissertation, Old Dominion University, Dept. of Mechanical Engineering, Norfolk, VA, December, 1995. 
${ }^{18}$ Lachowicz, J,T., Chokani, N., and Wilkinson, S.P., "Boundary-Layer Stability Measurements in a Hypersonic Quiet Tunnel”, AIAA Journal, Vol. 34, No. 12, December 1996.

${ }^{19}$ Shu, C.W., Essentially Non-Oscillatory And Weighted Essentially Non-Oscillatory Schemes For Hyperbolic Conservation Laws, NASA/CR-97-206253 and ICASE Report No. 97-6.

${ }^{20}$ Atkins, H.L., "High-Order ENO Methods for the Unsteady Compressible Navier-Stokes Equations," AIAA Paper 91-1557, 1991.

${ }^{21}$ Balakumar, P., Zhao, H., and Atkins, H., "Stability of Hypersonic Boundary Layers Over a Compression Corner," AIAA Paper 2002-2848, June 2002.

${ }^{22}$ Balakumar, P., and Malik, M.R., Effect of adverse Pressure Gradient and Wall Cooling on Instability of Hypersonic Boundary Layers, High Technology Corporation, Report No. HTC-9404, March 1994.

${ }^{23}$ Zhong, X., "Receptivity of Mach 6 Flow Over a Flared Cone to Freestream Disturbance," AIAA Paper 2004-253, January 2004. 


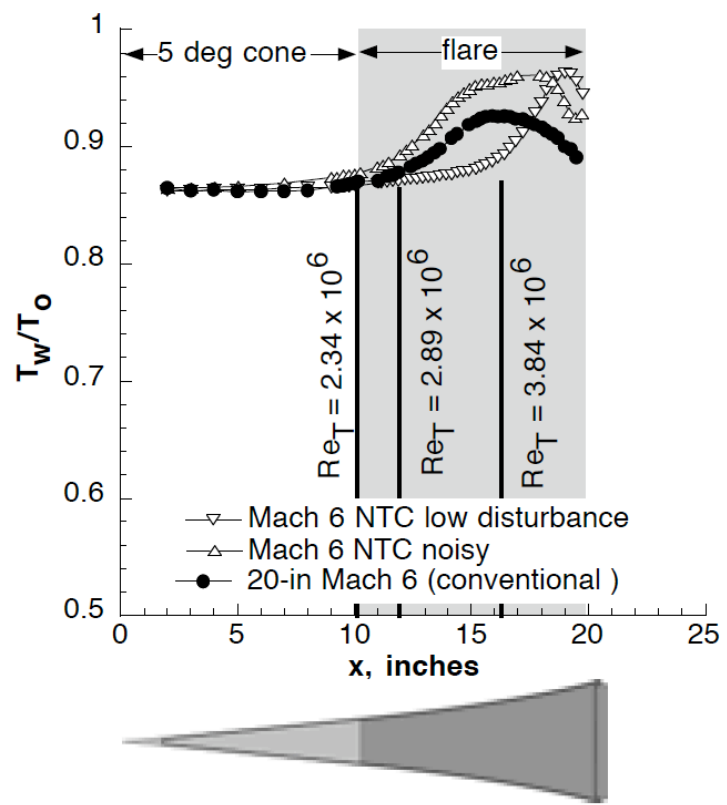

Figure 1. Comparison of smooth body transition onset locations over a flared cone obtained in the NASA LaRC Mach 6 Quiet Tunnel (at low and high freestream noise levels) and in the NASA LaRC 20 Inch Mach 6 Tunnel (Horvath, et al. ${ }^{10}$ ).

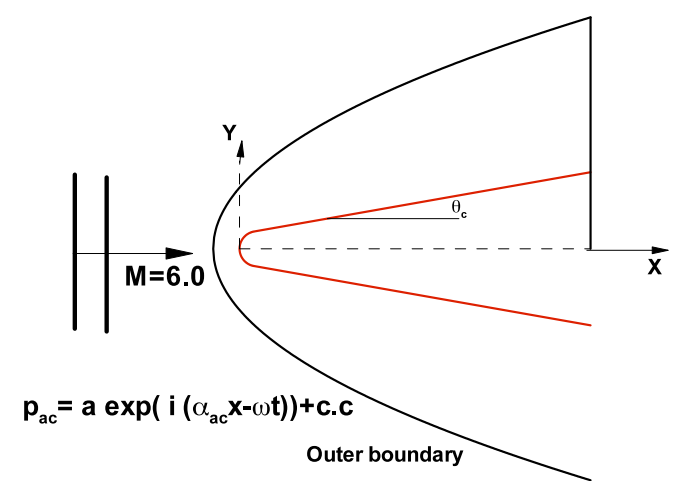

$M_{\infty}=6.0$

$\operatorname{Re}=10.4^{*} 10^{6} / \mathrm{m}$

$\mathrm{T}_{\infty}=52.43^{\circ} \mathrm{K}$

$\mathrm{T}_{\text {wall }}=300.0^{\circ} \mathrm{K}$

Leading edge radius $r_{0}=0.0125 \mathrm{~mm}$

Cone half-angle $=7$ degrees.

Figure 2. Schematic diagram of the computational model for flow over a 7-degree sharp-tipped cone.

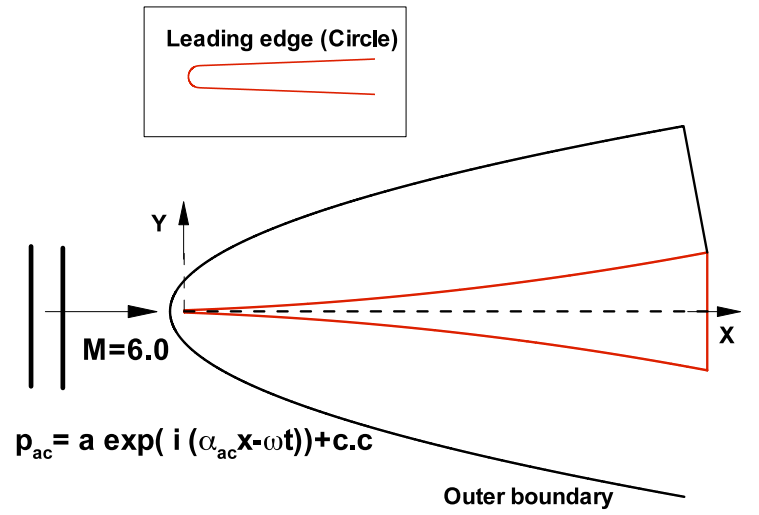

$M_{\infty}=6.0$

$\operatorname{Re}=10.3^{*} 10^{6} / \mathrm{m}$

$\mathrm{T}_{\infty}=52.80^{\circ} \mathrm{K}$

$\mathrm{T}_{\text {wall }}=300.0^{\circ} \mathrm{K}$

Leading edge radius $r_{0}=1.0 \mathrm{~mm}$

Flare is a circle with $3.0 \mathrm{~m}$ radius

Slope is 2 degrees at the joint

Figure 3. Schematic diagram of the computational model for flow over a blunt-tipped circular flared cone. 


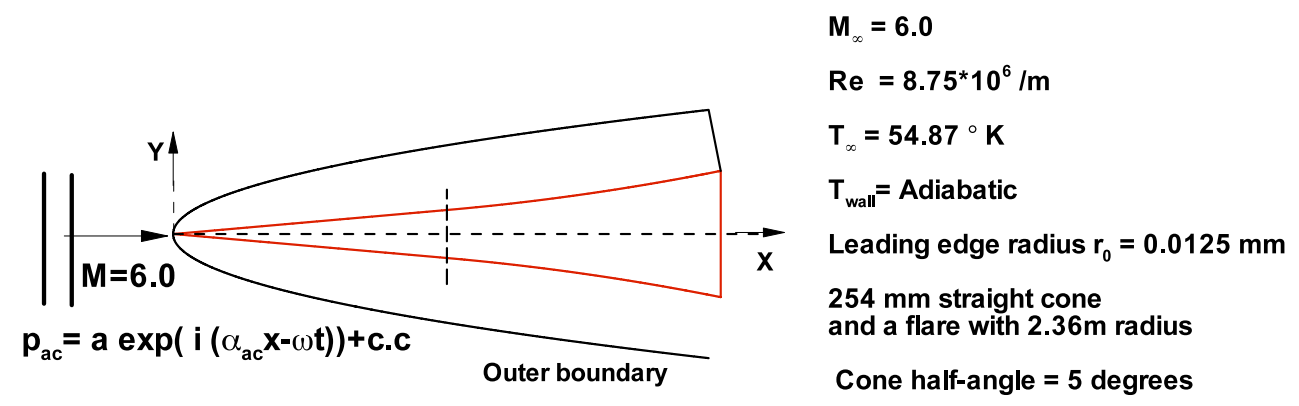

Figure 4. Schematic diagram of the computational model for flow over a sharp-tipped circular flared cone

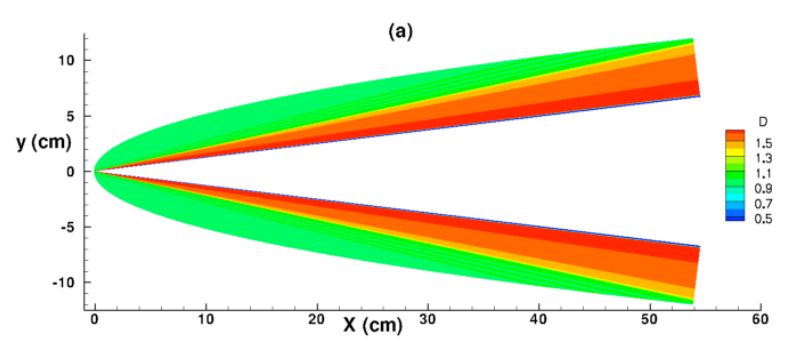

(b)

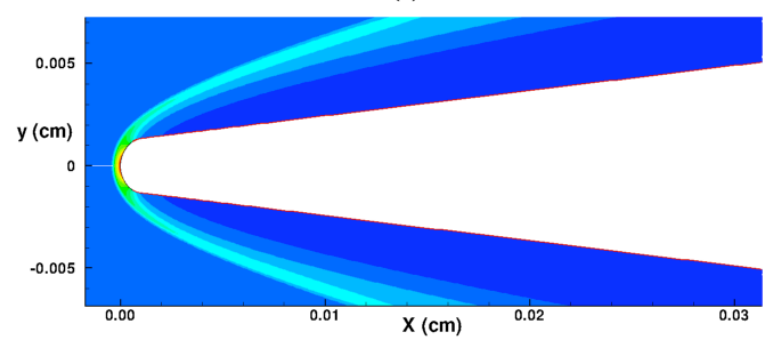

Figure 5. Mean density contours for flow over a 7-degree sharp-tipped cone at Mach 6.
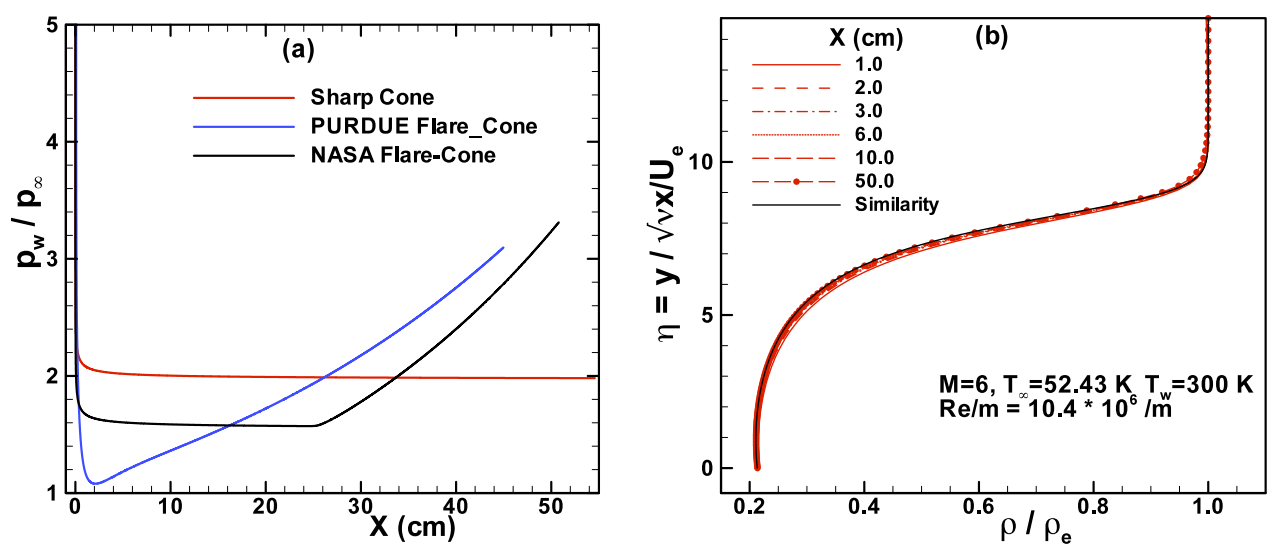

Figure 6. (a) Surface pressure distributions for the three model cases and (b) boundary layer density profiles at different axial locations for the sharp-tipped cone. 


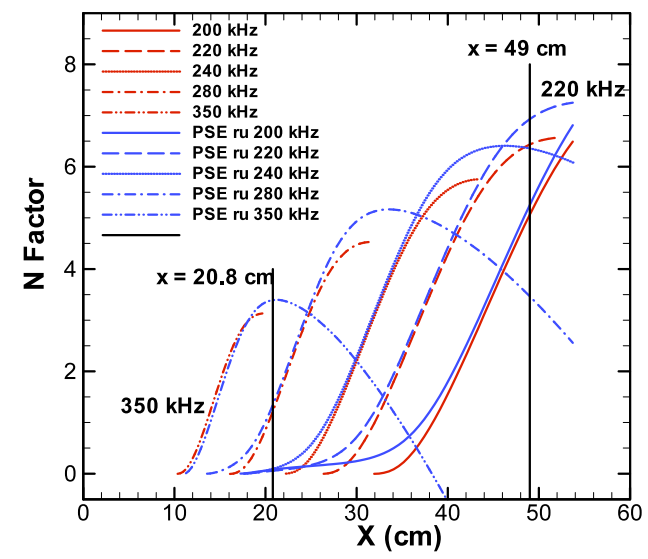

Figure 7. N-Factor curves computed from the linear stability analysis and the PSE analysis based on ( $\rho$ u $)_{\max }$ for the sharp-tipped cone.

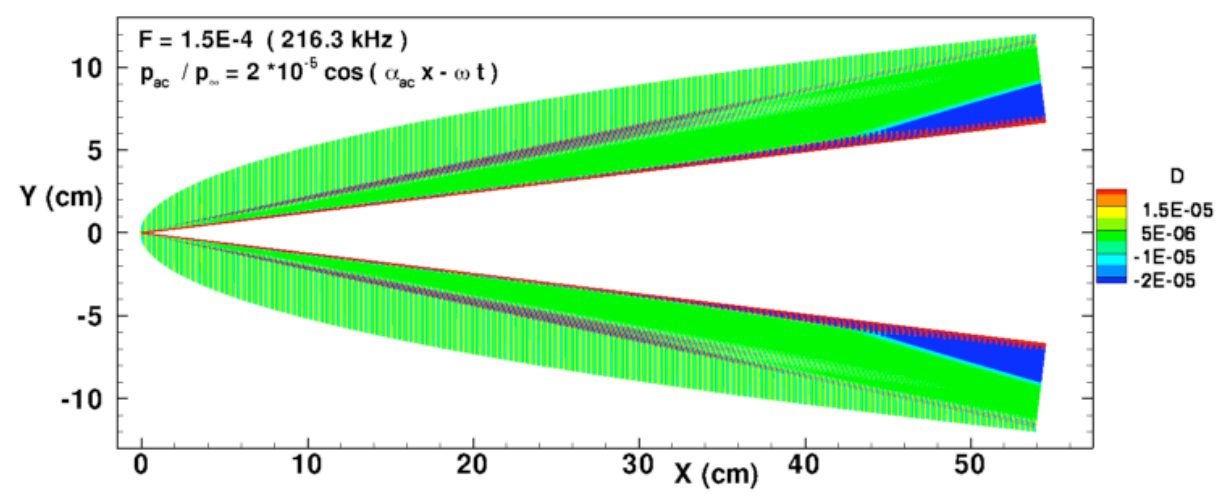

Figure 8. Density fluctuations generated by the interaction of a slow two-dimensional acoustic wave with the sharp-tipped cone.
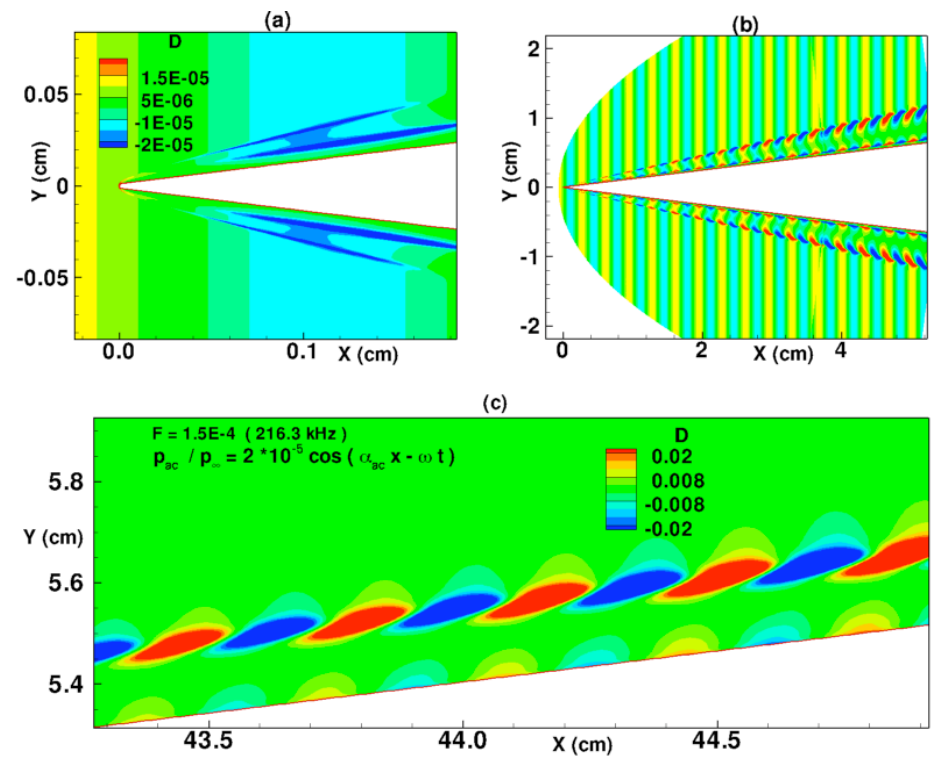

Figure 9. Density fluctuations generated by the interaction of a slow two-dimensional acoustic wave with the sharp-tipped cone (a) near the nose region, (b) near the tip of the cone, and (c) further downstream. 

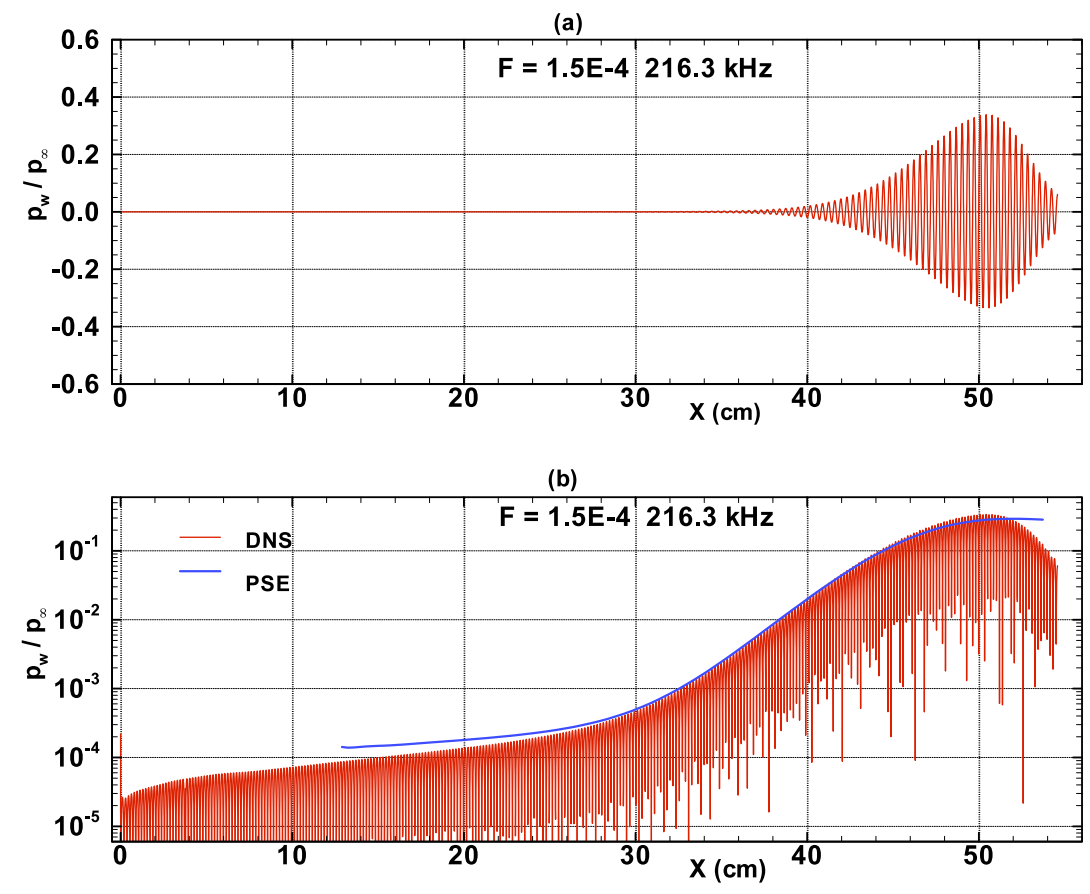

Figure 10. Wall-pressure fluctuations along the sharp-tipped cone obtained from the DNS for a slow acoustic wave. The results of the PSE calculations are shown for comparison.

(a) $X=208 \mathrm{~mm}$

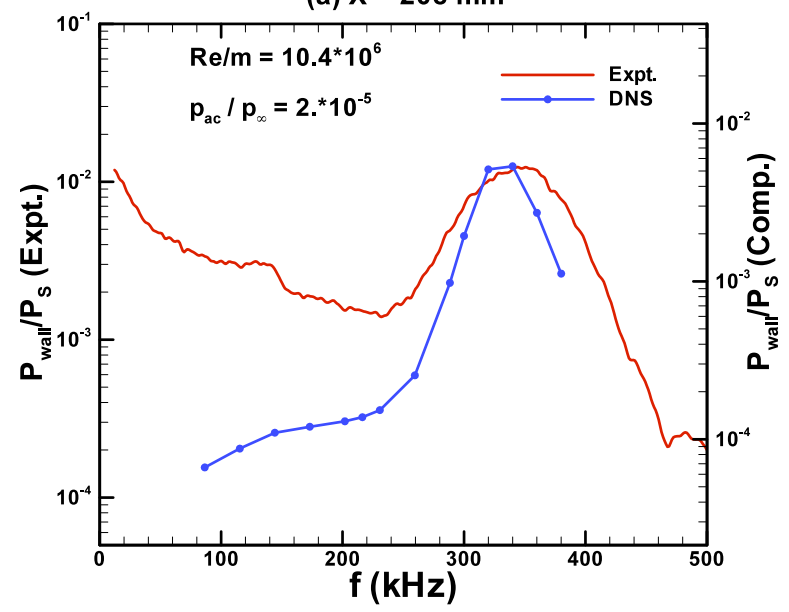

(b) $X=490 \mathrm{~mm}$

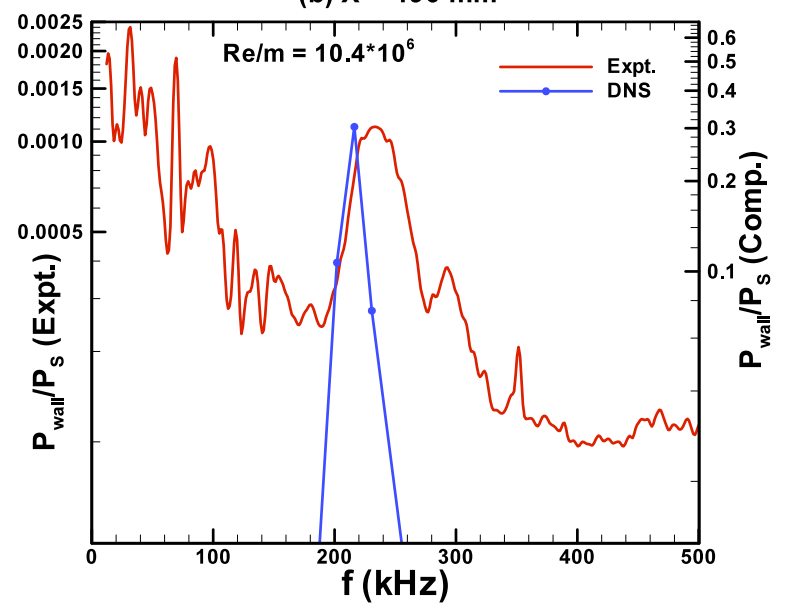

Figure 11. Comparison between the computed and measured wall-pressure spectrum for the sharp-tipped cone at a) $x=208 \mathrm{~mm}$ and b) $x=490 \mathrm{~mm}$. Experimental data from Casper et. al. ${ }^{16}$. 
(b)
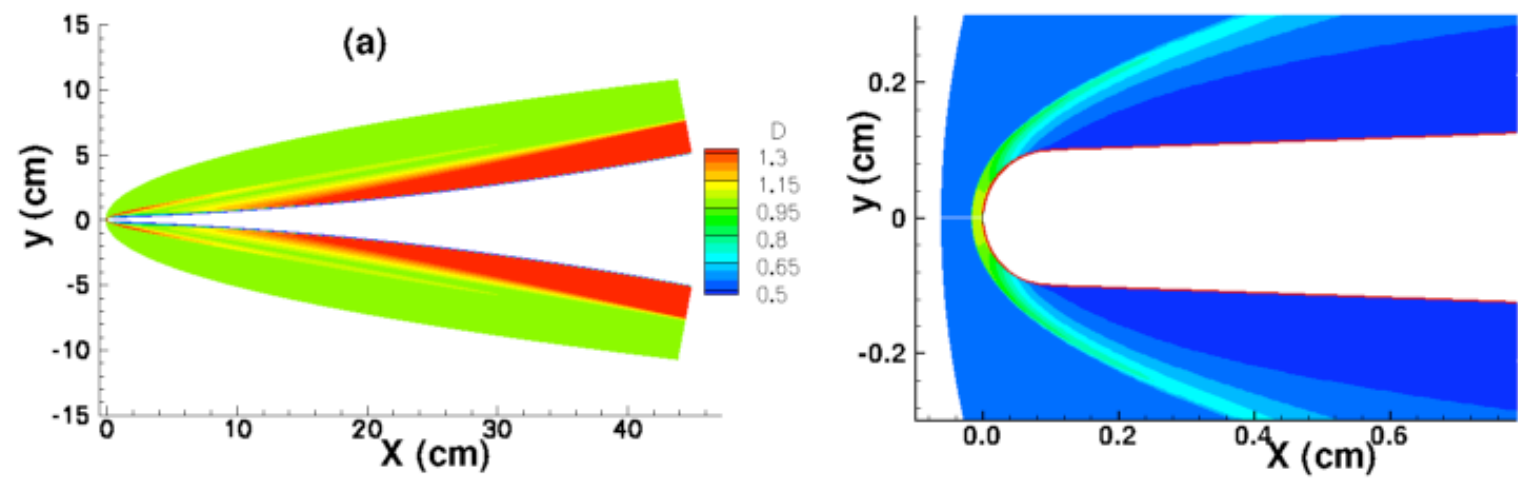

Figure 12. Mean density contours for flow over a circular flared cone with a blunt tip at Mach 6.
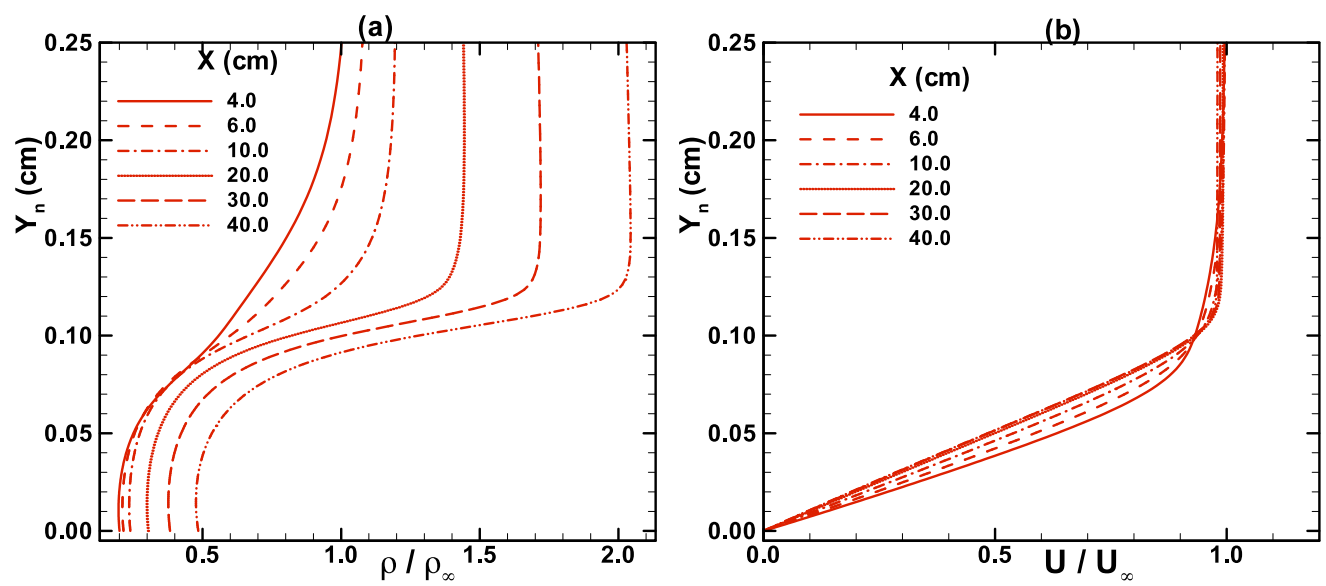

Figure 13. Boundary layer (a) density and (b) velocity profiles at different axial locations for the circular flared cone.
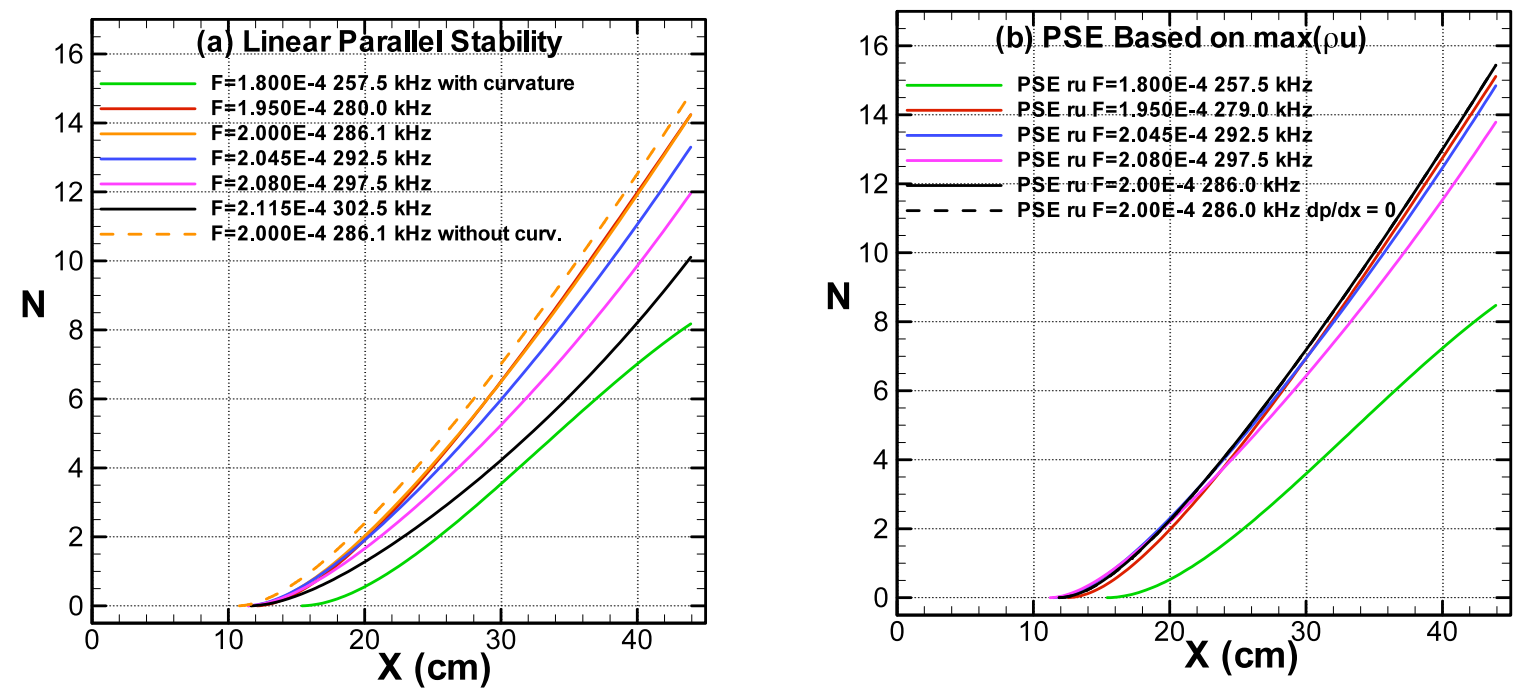

Figure 14. N-Factors computed from a) local linear stability analysis and b) non-local PSE calculations on the boundary layer profiles of the circular flared cone.

American Institute of Aeronautics and Astronautics 

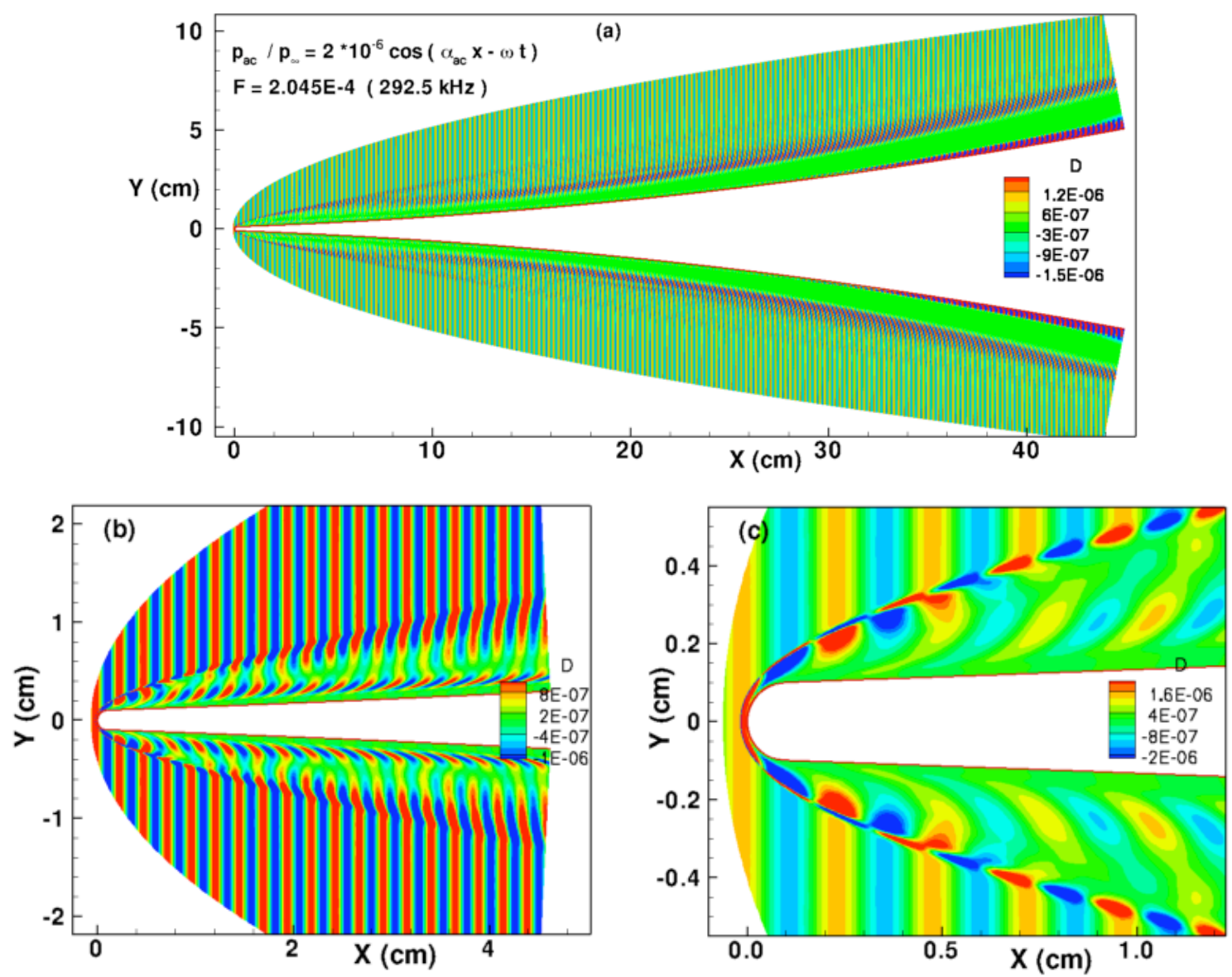

Figure 15. Density fluctuations generated by the interaction of a slow two-dimensional acoustic wave with the circular flared cone.
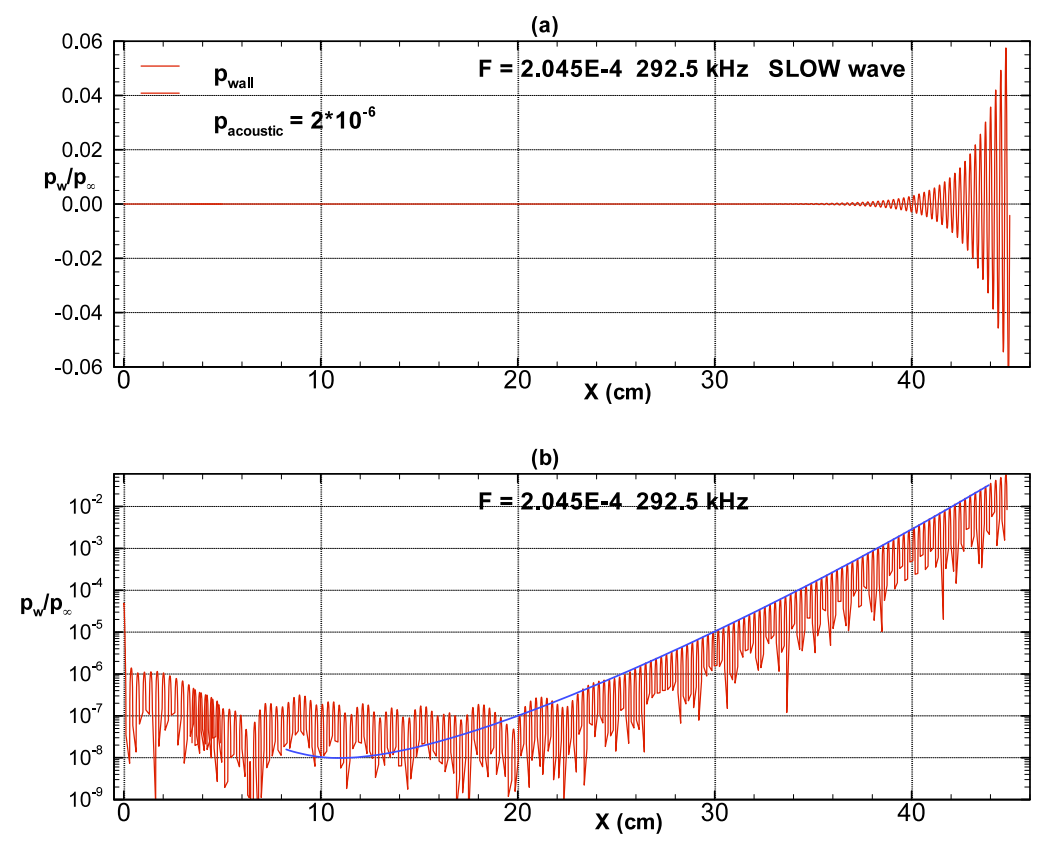

Figure 16. Wall-pressure fluctuations along the circular flared cone obtained from the DNS for a slow acoustic wave. The results of the PSE calculations are shown for comparison. 

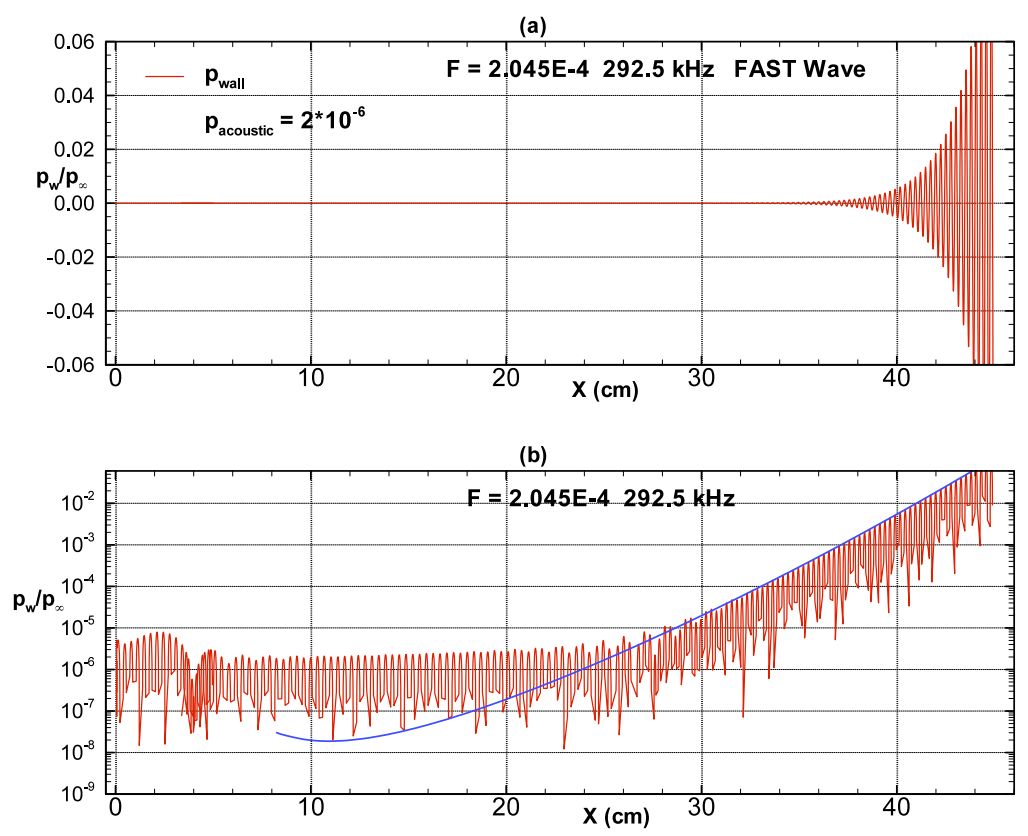

Figure 17. Wall-pressure fluctuations along the circular flared cone obtained from the DNS for a fast acoustic wave. The results of the PSE calculations are shown for comparison.

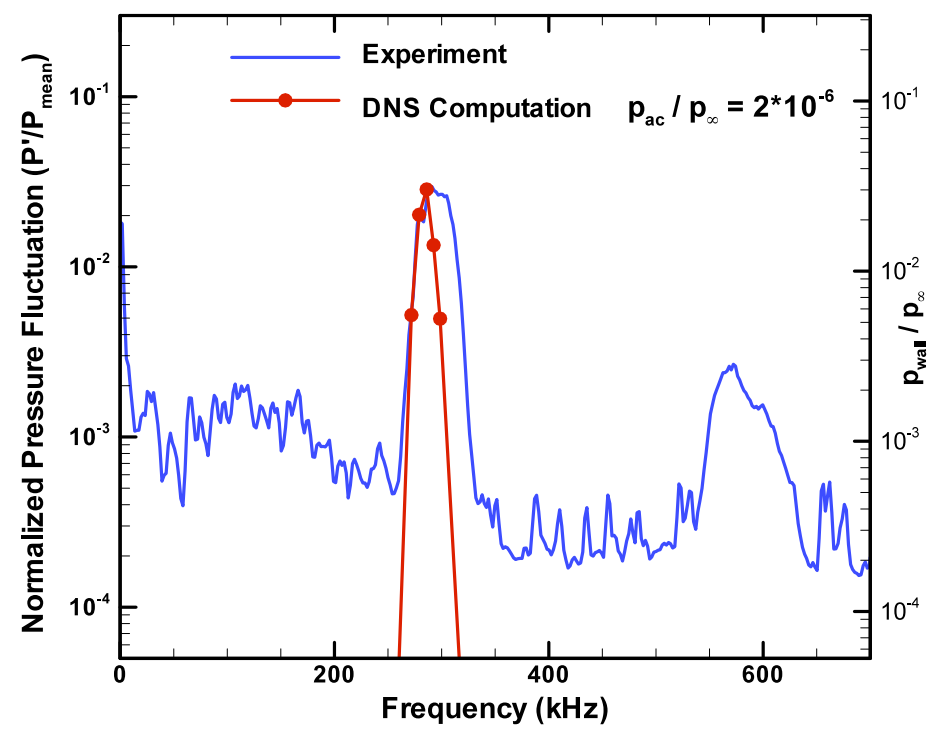

Figure 18. Comparison between the computed and measured wall-pressure spectrum for the circular flared cone at an axial location of $x=0.40 \mathrm{~m}$. Experimental data from Wheaton et. al. ${ }^{15}$. 


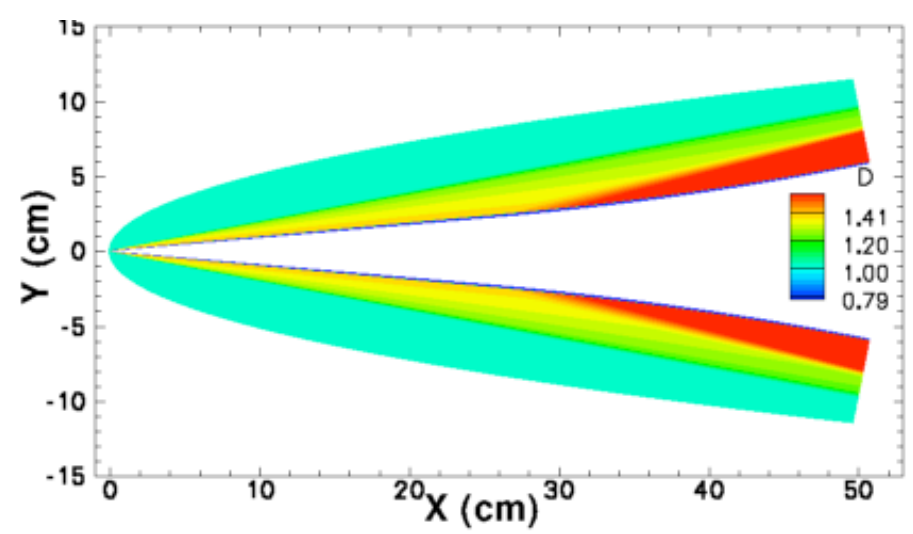

$\mathbf{M}=6$

Stagnation Pressure $=130$ psi

Stagnation Temperature $=450 \mathrm{~K}$

Reynolds number $=8.95^{\star} 10^{6} / \mathrm{m}$

Cone half-angle $=\mathbf{5}$ deg

Nose radius $=0.0005$ in .

Wall condition $=$ Adiabatic

Figure 19. Mean density contours for flow over a circular flared cone with a sharp tip at Mach 6.
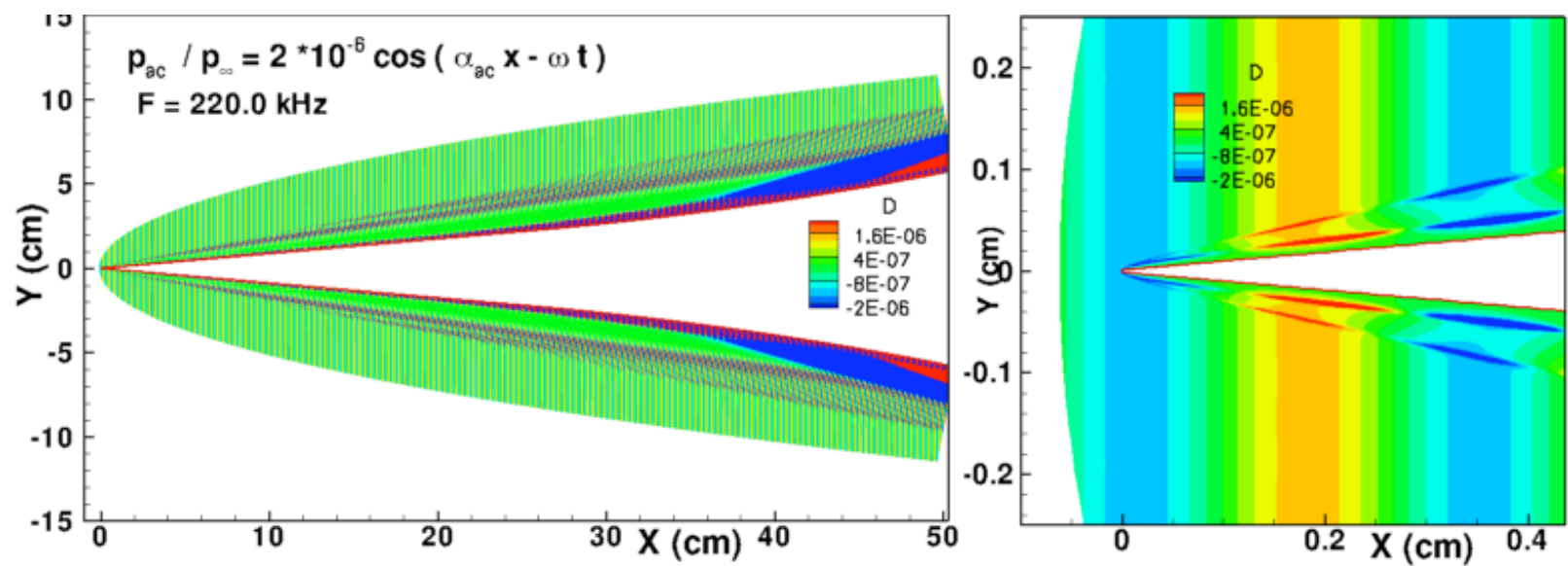

Figure 20. Density fluctuations generated by the interaction of a slow two-dimensional acoustic wave with the sharp-tipped flared cone.

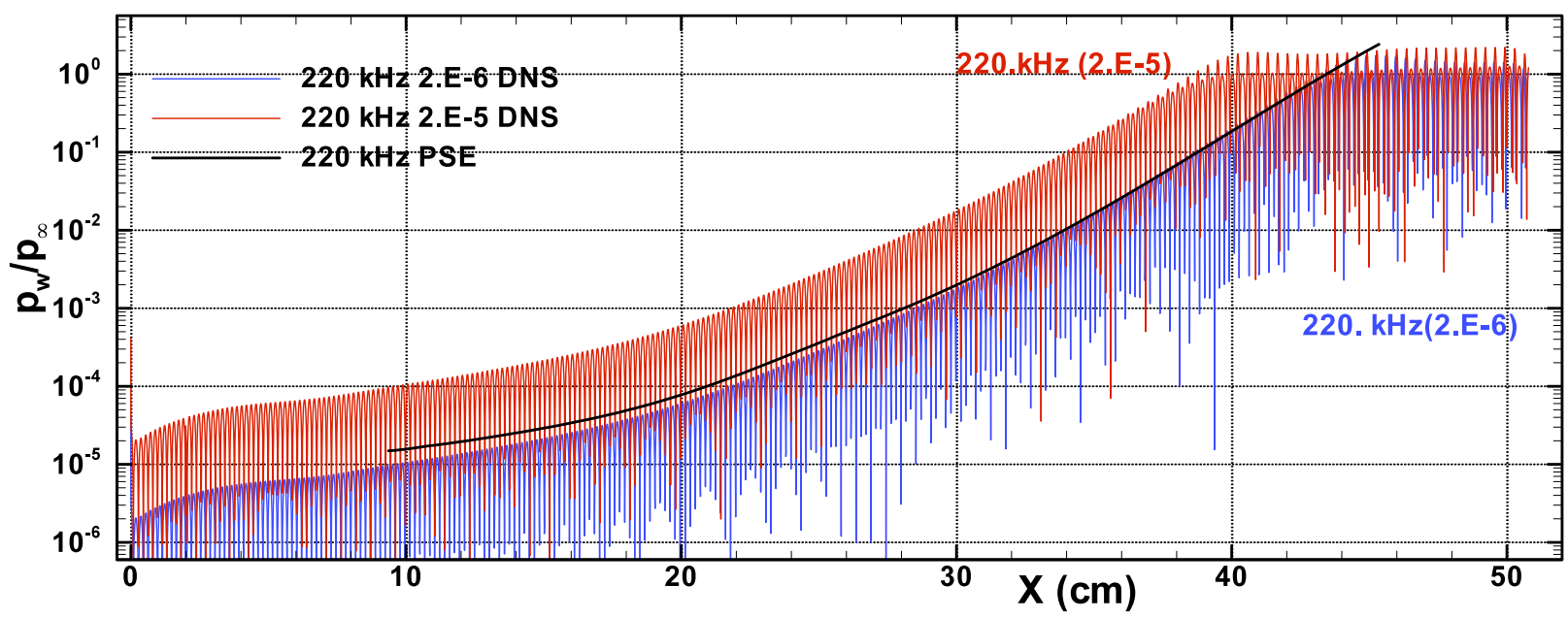

Figure 21. Wall-pressure fluctuations along the sharp-tipped flared cone obtained from the DNS for a slow acoustic wave with frequency $f=220 \mathrm{kHz}$. 


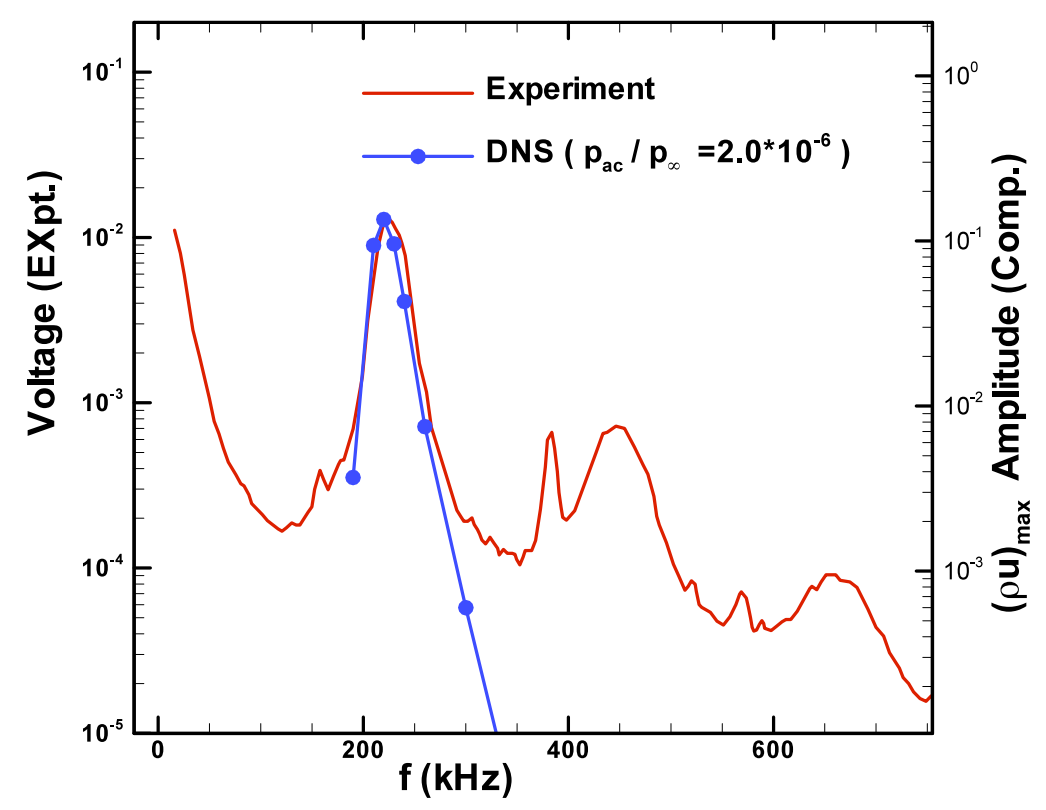

Figure 22. Comparison between the computed wall-pressure spectrum and a measured hot-wire voltage spectrum $^{18}$ for the sharp-tipped flared cone at an axial location of $x=40 \mathrm{~cm}$. Experimental data from Lachowicz et. al. ${ }^{18}$. 